\title{
Review of: "Effects of neoadjuvant chemotherapy in ovarian cancer patients with different germline BRCA1/2 mutational status: A retrospective study"
}

\author{
$\mathrm{Li} \mathrm{Li}^{1}$ \\ 1 Guangxi Medical University
}

Potential competing interests: The author(s) declared that no potential competing interests exist.

The objective of this study was to evaluate whether NAC followed by IDS against PDS has a differential effect on prognosis due to germline BRCA1/2 mutations. This study is dealing with clinically interesting topic has guiding significance for clinical work, and I suggest accepting this study, however, some issues need to be addressed before acceptance.

Methods

Page 6, line 13

1. All patients with NAC received 1-3 cycles of platinum-containing (carboplatin, cisplatin, or nedaplatin) chemotherapy, however, in Table1, the chemotherapy regimens of several patients were unknown, please explain the reason. In addition, please explain Multiple Platinum in Talbe1 in detail.

Page 7 , line 2

2. What was the 5-class classification standard? Please describe it if possible.

Results

Page 7, line 29

3. BRCAm was confirmed in $35.6 \%$ (108/308) of the included patients. Compared

to conventional reports, it is very high frequency. Of these, variants of VUS should be reported separately, clearly indicating a lack of adequate clinical evidence. In this study, how did you handle the patients with VUS?

4. This article studied germline BRCA mutation status, why not included somatic BRCA mutation status? Page 8, line 32

5. "ca125 above $1000 \mathrm{u} / \mathrm{ml}$ " should be "CA125 above $1000 \mathrm{U} / \mathrm{mL}$ "

Page 9, line9

6. COX analysis was performed on the factors affecting PFS in BRCAmut patients, but OS was not discussed. Tablel mentioned the use of PARP inhibitors in BRCAmut patients, which should be included in the analysis to explore whether the factors affecting OS are related to PARP inhibitors. 


\section{Tables \& Figrues}

7. About the appearance of Figure 2-3, it had better put the explanatuion of the patients analyzed on the Figures. For example, A of Figure 2 should be written as "all patients", B "BRCAmut patients", C "BRCAwt patients".

8. Please explain the NA in the tables and why statistical calculation was not performed.

9. Whether the value of Unknown in the tables has been calculated in the chi-square test? Please explain in detail. 